\title{
Adding possibilistic knowledge to probabilities makes many problems algorithmically decidable
}

\author{
Olga Kosheleva and Vladik Kreinovich \\ University of Texas at El Paso
}

\begin{abstract}
Many physical theories accurately predict which events are possible and which are not, or - in situations where probabilistic (e.g., quantum) effects are important - predict the probabilities of different possible outcomes. At first glance, it may seem that this probabilistic information is all we need. We show, however, that to adequately describe physicists' reasoning, it is important to also take into account additional knowledge - about what is possible and what is not. We show that this knowledge can be described in terms of possibility theory, and that the presence of this knowledge makes many problems algorithmically decidable.
\end{abstract}

Keywords: possibility theory, possibilistic knowledge, physicists' intuition, algorithmically decidable

\section{Need to Supplement Probabilistic Predictions with Possibilistic Information}

How physicists make their conclusions: why probabilities are sometimes not enough. Modern physics makes many very accurate predictions of different events. In situations when quantum effects are important and thus, deterministic predictions are not possible, physics predicts probabilities of different events; see, e.g., [5]. There are still many problems where we cannot accurately predict the events and/or their probabilities, but in many other situations, the accuracy of predictions is truly amazing.

At first glance, once we know the probabilities, we are done: we can thus predict the frequencies with which the corresponding events will occur in real life. In many situations, probabilities are indeed all we need. For example, when we predict that the probability of a coin falling heads is $1 / 2$, this means that in half of the cases, the coin will fall heads, in half, tails, and there is no other information that we can extract from observing the results of an actual coin toss: these results should be random.

This is true not only for coin tossing, but also for other predictions in which the predicted probability is "reasonable", i.e., not too small and not too close to 1 . However, the situation is somewhat different when it comes to events with a very small probability. Let us give a few simple examples.
According to statistical physics, entropy of a closed system can only increase. This means, for example, that if we place a cold kettle on a cold stove, it is not possible that the kettle will start boiling by itself, while the stove will get colder - although this transfer of energy from the stove to the kettle does not contradict to the energy conservation law.

How do physicists conclude that this is not possible? They estimate the probability of such an event and conclude that this probability is extremely small. From the purely mathematical viewpoint, the fact that this probability is not zero means that if we wait long enough, then we will still see a kettle boiling on a cold stove. However, this is not what the physicists claim. What they claim is that the kettle cannot boil. In other words, they claim that the corresponding event is simply not possible $[2,4,5]$.

Another example is the impossibility of spontaneous human levitation. The fact that a body has a non-zero temperature means that all the atoms and all the molecules in the body are randomly oscillating. Again, since all the molecules are going in random directions, there is a non-zero probability that they will all go into the same direction and a person will be spontaneously lifted above ground. What the physicists claim is not that such a possibility is rare, they claim is that it is simply not possible.

Physicists make similar conclusions about all irreversible events. For example, if we place a gas in one half of the box and leave another half - separated by a door - empty, then, when we open the door, gas will spread evenly through both halves of the box. From the purely mathematical viewpoint, it is also possible that, vice versa, if we start with a gas which is uniformly spread through the box, then at some future moment of time, all the molecules will concentrate in one half of this box, while the other half will remain empty: the probability of this event is small but still positive. However, what the physicists claim is that such a spontaneous separation is simply not possible.

Need to go beyond probabilities is in good accordance with common sense. The impossibility of events with very low probability may sound counter-intuitive, but it is actually in good accordance with common sense. Suppose that you flip a coin - which you believe to be fair - several times, and every time it falls heads. If this happens two, 
three, even ten times in a row, you may still continue believing that the coin is fair and that the actual probability of heads is indeed $1 / 2$. However, what if this happens 30 times in a row? 100 times in a row? Different people may have different thresholds, but for any person, there is some number after which the person will be absolutely sure that this coin is not fair.

Let us give another example. In each state lottery, usually, someone wins the big prize. If the same person wins the big prize two years in a row, one may still claim that this was a random coincidence. But what if the same person wins three years in a row? four years in a row? No matter how much you originally believe in the fairness of the state lottery process, if this continues year after year, eventually, every person will be convinced that the lottery is rigged.

Events with very small probability are not possible: can we describe this physical idea in purely probabilistic terms? We have mentioned that both physics and common sense use a rule that events with very small probability cannot happen. How can we describe this rule in precise terms?

At first glance, it may seem that we can describe this rule in purely probabilistic terms: namely, we can set up some threshold small value $p_{0} \ll 1$, and we can claim that any event with probability $\leq p_{0}$ is not possible. However, a simple example of coin tossing shows that proposal does not work. Indeed, what we want to claim is that after tossing a coin a large number $(N)$ of times, we cannot have a sequence HH. .. H of all heads. The probability of this event is $2^{-N}$, which, for large $N$, is indeed a very small number. So, at first glance, it may seem that if we take $p_{0} \geq 2^{-N}$, then we will be able to make the desired conclusion.

But the situation is not so easy. The problem is that any sequence of $N$ heads and tails - including the actual sequence that we will get after tossing a coin $N$ times - has the exact same probability $2^{-N}$. So, if we require that no event with probability $\leq p_{0}$ is possible, we come up with a strange conclusion that no sequence of heads and tails is possible at all - which makes no sense, since, of course, we can flip the coin $N$ times and record the results.

Comment. It is worth mentioning that there is a direct relation between this discussion and the notions of Algorithmic Information Theory, such as algorithmic randomness and Kolmogorov complexity; see, e.g., [17]. The main difference, however, is that the notion of algorithmic randomness is based on the assumption that events with probability 0 cannot occur, while we are trying to describe a more general statement: that not only events with zero probability cannot occur, but events with a sufficiently small positive probability cannot occur either.
Possibilistic information is needed. The above simple example shows that we cannot separate possible from impossible events by only using the known probabilities of different events. Thus, to properly describe physicists' reasoning (and our common sense), we need to supplement the probabilistic information with an additional information about what is possible.

\section{How to Describe the Desired Expert Information: Reminder}

What is possible: how to describe the opinion of a single expert. Let us start by describing a single expert's opinion on what is possible and what is not. Let $U$ be the universe of discourse, i.e., in our case, the set of possible events. We assume that we know the probabilities of different events, i.e., that for some subsets $S \subseteq U$, we know the probability $p(S)$ that the actual event will be in $S$.

From all possible events, the expert select a subset $T$ of all events which are possible. The main idea that we want to formalize is that if the probability is very small, then the corresponding event is not possible. What constitutes "very small" depends on the situation, but it is clear that if we have a definable sequence of events $A_{1} \supseteq A_{2} \supseteq \ldots \supset A_{n} \supseteq \ldots$, with $p\left(A_{n}\right) \rightarrow 0$, then for some sufficiently large $N$, the probability of the corresponding event $A_{N}$ becomes so small that this event is impossible, i.e., $T \cap A_{N}=\emptyset$.

This is what we trying to describe for the case of coin tossing: $A_{n}$ is the event when the heads appear in the first $n$ coin tosses; then, $p\left(A_{n}\right)=2^{-n} \rightarrow 0$.

In general, we arrive at the following formalization:

Definition 1. [8] Let $U$ be a set with a probability measure $p$. We say that a subset $T \subseteq U$ is a set of possible elements if for every definable sequence $A_{n}$ for which $A_{n} \supseteq A_{n+1}$ and $p\left(A_{n}\right) \rightarrow 0$, there exists $N$ for which $T \cap A_{N}=\emptyset$.

Need to go beyond probabilities. Sometimes, physicists use similar arguments even in situations when we do not know the probabilities. For example, physicists often expand a dependence in Taylor series $f(x)=a_{0}+a_{1} \cdot x+a_{2} \cdot x^{2}+\ldots$ When $x$ is small, i.e., when $|x| \leq \delta$ for some small $\delta$, they argue that we can safely ignore quadratic (and higher order) terms in this expansion and assume that $f(x) \approx a_{0}+a_{1} \cdot x$; see, e.g., [5].

This conclusion is definitely justified if we know the value $a_{2}$, or, at least, if we know some a priori bound $C$ on this value. Then, $\left|a_{2} \cdot x^{2}\right| \leq C \cdot \delta^{2}$, so when $\delta$ is sufficiently small, this term can indeed be safely ignored. However, physicists make this conclusion even when we do not know of any a priori bound on $a_{2}$. Their idea is that values which are too large are highly improbable.

In this case, we also have a series of events $A_{n} \supseteq$ $A_{n+1}$ : namely, $A_{n}$ is the set of situations in which 
$\left|a_{2}\right|>n$. Here, we do not have probabilities, but we know that $\cap A_{n}=\emptyset$. Thus, no matter what is the (unknown) probability measure $p$, we have $p\left(A_{n}\right) \rightarrow 0$. As a result, we can use Definition 1 and conclude that for a sufficiently large $N$, events from $A_{N}$ are impossible - hence $\left|a_{2}\right| \leq N$.

Such situations lead to the following alternative definition that can be even when we do not know probabilities; see, e.g., [6, 7, 8, 10, 11, 12, 13, 14, 15, 16]:

Definition 2. Let $U$ be a set. We say that a subset $T \subseteq U$ is a set of possible elements if for every definable sequence $A_{n}$ for which $A_{n} \supseteq A_{n+1}$ and $\cap A_{n}=\emptyset$, there exists $N$ for which $T \cap A_{N}=\emptyset$.

What is known and we plan to prove. It is known (see, e.g., [12]) that if we restrict ourselves to possible elements, then some problems become algorithmically solvable (see Section 4 for more detail). In Section 5 of this paper, we extend this result to a general class of problems.

We also show, in Section 3, how the above definitions relate to the usual notions of possibility theory; see, e.g., [3, 22].

\section{Relation to Possibility Theory}

From individual experts to groups of experts. Reasoning in physics does not rely on the opinion of a single expert: physics is versatile, and it is important to have several experts to cover all possible topics. Let $E$ denote the set of all the experts.

Experts, in general, may have somewhat different ideas on what is possible and what is not. As a result, instead of a single set $T$ of possible events, we have several different sets $T_{e}$ corresponding to different experts $e$.

How to reformulate this information in terms of the usual possibility theory. Instead of describing, for each expert, which events are possible or not possible according to this expert, it makes sense to instead describe, for each event $s$, which experts consider this possible possible and which not possible. In this case, to each event $s$, we put into correspondence the set $m(s) \subseteq E$ of all the experts who believe that $s$ is possible.

One can easily show that this description is equivalent to the description in terms of the sets $T_{e}$. Indeed:

- if we know all the sets $T_{e}$, then for each event $s$, we can assign the set $m(s)=\left\{e: s \in T_{e}\right\}$;

- vice versa, if we know all the values $m(s)$, then, to each expert $e$, we can assign the set

$$
T_{e}=\{s: e \in m(s)\} .
$$

In addition to describing which individual events are possible, it makes sense to describe which sets of events are possible. For example, we can ask for the possibility that we have one particle, or two particles, or three particles, and we can also ask for the possibility that we have at least one particle.

In precise terms, instead of a single event $s \in U$, we have a set of events $S \subseteq U$, and we are interested in checking when some event from $S$ is possible. Let $m(S)$ denote the set of all the experts who believe that one of the events $s \in S$ is possible. An expert believes that some event $s \in S$ is possible if and only if for some $s \in S$, the expert believes that the corresponding event $s$ is possible. Thus, the set $m(S)$ is equal to the union of all the sets $m(s)$ corresponding to different events $s \in S$ :

$$
m(S)=\bigcup_{s \in S} m(s) .
$$

Thus, for every two sets $S$ and $S^{\prime}$, we have

$$
m\left(S \cup S^{\prime}\right)=m(S) \cup m\left(S^{\prime}\right) .
$$

On the class of all sets, there is a natural (partial) order $\leq$ : namely, the subset relation $S \subseteq S^{\prime}$. In terms of this relation, every two sets have the least upper bound (join) $S \vee S^{\prime}=S \cup S^{\prime}$, and the greatest lower bound (meet) $S \wedge S^{\prime}=S \cap S^{\prime}$. The whole class of sets with these two operations is thus a lattice. In terms of this lattice operations, we have

$$
m\left(S \cup S^{\prime}\right)=m(S) \vee m\left(S^{\prime}\right) .
$$

This is exactly how a possibility measure is defined in possibility theory [3] - except that usually, possibility measures have values in the interval $[0,1]$, while for our formalization, we need lattice-valued possibility measures.

What are the corresponding $\alpha$-cuts. Since we have shown that our description of possibility in physics can be reformulated in terms of possibility theory, and possibility theory is closely related to fuzzy [3, 22], in makes sense to analyze what fuzzy concepts (see, e.g., $[9,18,21]$ ) will look like in our case. One of such notions - actively used in fuzzy set theory, especially when processing fuzzy data is the notion of an $\alpha$-cut.

For each membership function $m(s)$ and for each degree $\alpha$, the $\alpha$-cut is defined as

$$
m^{\alpha}=\{s: m(s) \geq \alpha\} .
$$

In our case, degrees are subsets of the set $E$ of all the experts, and $\geq$ means a super set relation. Thus, $m(s) \geq \alpha$ means all experts from the set $\alpha$ believe that the event $s$ is possible.

In terms of the sets $T_{e}$, this means that

$$
m^{\alpha}=\bigcap_{e \in \alpha} T_{e}
$$

In particular, for $\alpha=\{e\}$, we have $m^{\alpha}=T_{e}$. Each set $T_{e}$ is a set of possible elements, and one can easily conclude, from Definitions 1 and 2, that any subset of such a set also has this property. Thus, 
not only every set $T_{e}$ is a set of possible elements, but for every $\alpha$, the corresponding $\alpha$-cut is a set of possible elements.

Vice versa, if for every $\alpha$, every $\alpha$-cut is a set of possible element, then, in particular, this is true for $\alpha=\{e\}$ for each $e \in E$; thus, in this case, every set $T_{e}$ is indeed a set of possible elements in the sense of Definitions 1 or 2 .

Thus, we get the following equivalent reformulation of our description of the physicists' possibilistic knowledge: this knowledge can be described as a lattice-valued possibility measure for which, for every $\alpha$, the corresponding $\alpha$-cut is a set of possible elements in the sense of Definition 1 or 2 .

\section{Many Problems Are Algorithmically Decidable If Restrict Ourselves to Possible Elements: What Is Known}

In general, many problems are not algorithmically decidable. In general, many computational problems are not algorithmically decidable; see, e.g., [1, 20]. As a simple example, let us consider the problem of deciding whether two given real numbers are equal or not.

In this problem, the input consists of two real numbers, and the desired output is "yes" or "no", depending on whether these numbers are equal or not.

To describe this problem in precise terms, we need to formulate how exactly we present the input to a computer. In practice, real numbers come from measurements, and measurements are never absolutely accurate. In principle, we can measure a real number $x$ with higher and higher accuracy (if not now, then in the future). For example, for any integer $n$, we can measure this number with the accuracy of $n$ binary digits, i.e., with the accuracy of $2^{-n}$. As a result of each such measurement, we get a rational number $r_{n}$ for which $\left|x-r_{n}\right| \leq 2^{-n}$. This is exactly the usual definition of a computable real number: it is a process (maybe algorithmic, maybe involving measurements) that enables us, given an integer $n$, to generate a rational number $r_{n}$ for which $\left|x-r_{n}\right| \leq 2^{-n}[1,20]$.

Computing with computable real numbers means that, in addition to usual computational steps, we can also generate some $n$, get the corresponding value $r_{n}$, and then use this value is computations.

Some things can be computed this way. For example, if we know computable real numbers $x$ and $y$, then their sum $z=x+y$ is also a computable real number. Indeed, to compute the $2^{-n_{-}}$ approximation $t_{n}$ to the $\operatorname{sum} z$, it is sufficient to take the sum $s_{n}=r_{n+1}+s_{n+1}$ of $2^{-(n+1)}$ approximations $r_{n+1}$ and $s_{n+1}$ to $x$ and $y$. Indeed, from $\left|x-r_{n+1}\right| \leq 2^{-(n+1)}$ and $\left|y-s_{n+1}\right| \leq 2^{-(n+1)}$, we can then conclude that

$$
\left|z-s_{n}\right|=\left|(x+y)-\left(x_{n+1}+y_{n+1}\right)\right|=
$$

$$
\begin{gathered}
\left|\left(x-x_{n+1}\right)+\left(y-s_{n+1}\right)\right| \leq \\
\left|x-x_{n+1}\right|+\left|y-s_{n+1}\right| \leq \\
2^{-(n+1)}+2^{-(n+1)}=2^{-n} .
\end{gathered}
$$

However, it is not possible to algorithmically check whether the two computable numbers $x$ and $y$ are equal or not. Indeed, if this was possible, then, e.g., for equal real numbers $x=y=0$ for which $r_{n}=s_{n}=0$ for all $n$, our procedure will return the answer "yes". This procedure consists of finitely many steps, thus it can only ask for finitely many values $r_{n}$ and $s_{n}$. Let $N$ be the smallest number which is larger than all the requests $n$. Then, we can keep the same $x$, but take instead a different $y^{\prime}=2^{-N} \neq 0$ for which $s_{1}^{\prime}=\ldots=s_{N-1}^{\prime}=0$ (so our equality-checking procedure will not notice the difference), but $s_{N}^{\prime}=s_{N+1}^{\prime}=\ldots=2^{-N}$. Since our procedure cannot notice the difference between $y$ and $y^{\prime}$, it will still produce the same answer - that yes, the inputs are equal - while in reality, the new inputs $x=0$ and $y^{\prime}=2^{-N} \neq 0$ are different.

Similar negative results are known for many other problems $[1,20]$.

If we restrict ourselves to possible pairs of real numbers, then equality becomes decidable. Let us show, following [12], that if we restrict ourselves to possible pairs $(x, y)$, then it is algorithmically possible to check whether $x=y$ or $x \neq y$.

Indeed, the fact that we consider possible pairs of real numbers means that on the set $U=\mathbb{R} \times \mathbb{R}$ of all possible pairs of real numbers, we have a subset $T$ of possible numbers that satisfied Definition 2 . In particular, we can consider the following definable sequence of sets

$$
A_{n} \stackrel{\text { def }}{=}\left\{(x, y): 0<|x-y| \leq 2^{-n}\right\} .
$$

One can easily see that $A_{n} \supseteq A_{n+1}$ for all $n$ and that $\cap A_{n}=\emptyset$. Thus, by Definition 2 , there exists a natural number $N$ for which $T \cap A_{N}=\emptyset$, i.e., for which no element $s \in T$ belongs to the set $A_{N}$. This, in turn, means that for every pair $(x, y) \in T$, either $|x-y|=0$ (i.e., $x=y$ ) or $|x-y|>2^{-N}$.

So, to check whether $x=y$ or not, it is sufficient to compute both $x$ and $y$ with accuracy $2^{-(N+2)}$, i.e., to compute values $r_{N+2}$ and $s_{N+2}$ for which $\left|x-r_{N+2}\right| \leq 2^{-(N+2)}$ and $\left|y-s_{N+2}\right| \leq 2^{-(N+2)}$. Then:

- if $x=y$, then, due to triangle inequality, we have

$$
\begin{gathered}
\left|r_{N+1}-s_{N+2}\right| \leq\left|x-r_{N+2}\right|+\left|x-s_{N+2}\right| \leq \\
2^{-(N+2)}+2^{-(N+2)}=2^{-(N+1)} ;
\end{gathered}
$$

- on the other hand, if $x \neq y$, then from $|x-y|>$ $2^{-N}$, we conclude that

$$
\begin{gathered}
\left|r_{N+1}-s_{N+2}\right| \geq \\
|x-y|-\left|x-r_{N+2}\right|-\left|y-s_{N+2}\right|>
\end{gathered}
$$




$$
\begin{gathered}
2^{-N}-\left(2^{-(N+2)}+2^{-(N+2)}\right)= \\
2^{-N}-2^{-(N+1)}=2^{-(N+1)} .
\end{gathered}
$$

Thus, by checking whether $\left|r_{N+1}-s_{N+2}\right| \leq 2^{-(N+1)}$ or whether $\left|r_{N+1}-s_{N+2}\right|>2^{-(N+1)}$, we can decide whether $x=y$ or $x \neq y$.

Here, we compare rational numbers, i.e., ratios of integers, and for rational numbers, we can indeed algorithmically tell whether one is smaller or the other one is smaller.

\section{Many Problems Are Algorithmically Decidable If Restrict Ourselves to Possible Elements: A New General Result}

Towards a general description of similar properties. To generalize the above result, let us come up with a general description of similar properties.

Let us start with reformulating the question of whether $x=y$ in generalizable terms. Specifically, we would like to describe the corresponding property in terms of the observable sequences $r_{n}$ and $s_{n}$ describing the real numbers $x$ and $y$.

The equality between real numbers can indeed be described in these terms. Indeed, if $x=y$, then, for every $n$, we have

$\left|r_{n}-s_{n}\right| \leq\left|r_{n}-x\right|+\left|x-s_{n}\right| \leq 2^{-n}+2^{-n}=2^{-(n-1)}$.

Vice versa, let us assume that we have two computable real numbers $x$ and $y$ for which $\left|r_{n}-s_{n}\right| \leq$ $2^{-(n-1)}$ for all $n$. In this case, due to $\left|x-r_{n}\right| \leq 2^{-n}$ and $\left|y-s_{n}\right| \leq 2^{-n}$, we have

$$
\begin{gathered}
|x-y| \leq\left|x-r_{n}\right|+\left|r_{n}-s_{n}\right|+\left|s_{n}-y\right| \leq \\
2^{-n}+2^{-(n-1)}+2^{-n}=2^{-(n-2)} .
\end{gathered}
$$

Since this hold for every $n$, for $n \rightarrow \infty$, we get $x=y$.

Thus, the equality between computable real numbers has the form

$$
\forall n\left(\left|r_{n}-s_{n}\right| \leq 2^{-(n-1)}\right) .
$$

In general, as shown, e.g., in [19, 20], many properties involving limits, differentiability, etc., can be described in similar terms, namely as an arithmetic formula

$$
Q n_{1} Q n_{2} \ldots Q n_{k} F\left(r_{1}, \ldots, r_{\ell}, n_{1}, \ldots, n_{k}\right),
$$

where:

- each $Q n_{i}$ is either a universal quantifier $\forall n_{i}$ or an existential quantifier $\exists n_{i}$,

- $r_{1}, \ldots, r_{\ell}$ are corresponding sequences, and

- the property $F$ is simply a propositional ("and", "or", and "not") combination of equalities and inequalities between the explicitly computable rational-valued expressions.
In the above example of checking whether two given real numbers are equal:

- we have two sequences $\ell=2$,

- we only have one quantifier $k=1$,

- this quantifier is a universal quantifier $Q_{1}=\forall$, and

- the property $F$ has (in these terms) the form

$$
\left|r_{1}\left(n_{1}\right)-r_{2}\left(n_{1}\right)\right| \leq 2^{-\left(n_{1}-1\right)} .
$$

Let us show that for all such arithmetic expressions, the presence of possibilistic information leads to algorithmic decidability.

Main Result. For every arithmetic expression (1), for every set $T$ of possible tuples $r=\left(r_{1}, \ldots, r_{\ell}\right)$, there exists an algorithm that, given a tuple $r=$ $\left(r_{1}, \ldots, r_{\ell}\right) \in T$, checks whether the formula (1) holds or not.

Proof. If the formula (1) had no quantifiers, then we could simply plug in the corresponding values into this formula and check whether the corresponding formula holds or not. The problem is with the quantifiers: while we can easily check whether some property holds for a specific value $n_{i}$, it is not possible to directly check whether this property holds for all infinitely many natural numbers $n_{i}=0,1,2, \ldots$ The situation would be different if we could have a bound $N$ on possible values of $n_{i}$, i.e., if the quantifier had the form $\forall n_{i} \leq N$ or $\exists n_{i} \leq N$ : in this case, we can simply test all possible values $n_{i} \leq N$.

Let us show that for tuples from the set $T$, we can indeed have such bounds on the variables $n_{i}$. Let us start with a bound on $n_{1}$. For the variable $n_{1}$, there are two possible cases: when $Q_{1}$ is a universal quantifier and when $Q_{1}$ is an existential quantifier. Let us consider these two cases one by one.

In the first case, the formula (1) has the form $\forall n_{1} G\left(n_{1}\right)$, for some expression $G\left(n_{1}\right)$ (with one fewer quantifier). Let us take

$$
A_{n}=\left\{r: \forall n_{1}\left(n_{1} \leq n \rightarrow G\left(n_{1}\right)\right) \& \neg \forall n_{1} G\left(n_{1}\right)\right\} .
$$

One can easily check that $A_{n} \supseteq A_{n+1}$ and $\cap A_{n}=\emptyset$. Thus, there exists a natural number $N$ for which $T \cap A_{N}=\emptyset$. So, for $r \in T$, if

$$
\forall n_{1}\left(n_{1} \leq N \rightarrow G\left(n_{1}\right)\right)
$$

we cannot have $\neg \forall n_{1} G\left(n_{1}\right)$, so we must have $\forall n_{1} G\left(n_{1}\right)$. Clearly, $\forall n_{1} G\left(n_{1}\right)$ always implies $\forall n_{1}\left(n_{1} \leq N \rightarrow G\left(n_{1}\right)\right)$. Thus, for $r \in T, \forall n_{1} G\left(n_{1}\right)$ with an unlimited quantifier is equivalent to a formula $\forall n_{1}\left(n_{1} \leq N \rightarrow G\left(n_{1}\right)\right)$ with a bounded quantifier.

In the second case, the formula (1) has the form $\exists n_{1} G\left(n_{1}\right)$, for some expression $G$ (with one fewer quantifier). Let us take

$$
A_{n}=\left\{r: \neg \exists n_{1}\left(n_{1} \leq n \& G\left(n_{1}\right)\right) \& \exists n_{1} G\left(n_{1}\right)\right\} .
$$


One can easily check that $A_{n} \supseteq A_{n+1}$ and $\cap A_{n}=\emptyset$. Thus, there exists a natural number $N$ for which $T \cap A_{N}=\emptyset$. So, for $r \in T$, if

$$
\neg \exists n_{1}\left(n_{1} \leq N \& G\left(n_{1}\right)\right),
$$

we cannot have $\exists n_{1} G\left(n_{1}\right)$, so we must have $\neg \exists n_{1} G\left(n_{1}\right)$. Clearly, $\neg \exists n_{1} G\left(n_{1}\right)$ always implies

$$
\neg \exists n_{1}\left(n_{1} \leq N \& G\left(n_{1}\right)\right) .
$$

Thus, for $r \in T, \neg \exists n_{1} G\left(n_{1}\right)$ is equivalent to

$$
\neg \exists n_{1}\left(n_{1} \leq N \& G\left(n_{1}\right)\right) .
$$

So, by taking negations, we conclude that the original formula $\exists n_{1} G\left(n_{1}\right)$ with an unlimited quantifier is equivalent to a formula

$$
\exists n_{1}\left(n_{1} \leq N \& G\left(n_{1}\right)\right)
$$

with a bounded quantifier.

Now, we have reduced the original formula with $k$ quantifiers to a formula in which the first quantifier is bounded. This bounded-quantifier formula is equivalent to, correspondingly,

$$
G(0) \& G(1) \& \ldots \& G(N)
$$

or to

$$
G(0) \vee G(1) \vee \ldots \vee G(N),
$$

where the corresponding formulas $G\left(n_{1}\right)$ have $k-1$ quantifiers. So, if we can find the truth values of each of these (finitely many) formulas $G\left(n_{1}\right)$, we could be able to check the truth value of the original formula (1).

For each of these formulas $G\left(n_{1}\right)$ with $k-1$ quantifiers, we can apply the same reduction to reduce them to formulas with $k-2$ quantifiers, etc., until we get formulas with no quantifiers at all - which can be therefore directly checked.

This reduction proves that it is indeed algorithmically possible to check whether a given formula (1) holds or not for a given tuple $r$. The main result is proven.

\section{Conclusions}

In this paper, we show that in order to adequately describe physical reasoning, we need to supplement the usual probabilistic information with an additional knowledge describing what is possible and what is not. We show that a natural description of this additional knowledge is closely related to possibility theory. It turns out that in the presence of this additional knowledge, many physics-related problems become algorithmically decidable.

\section{Acknowledgments}

This work was supported in part by the National Science Foundation grants HRD-0734825 and HRD1242122 (Cyber-ShARE Center of Excellence) and DUE-0926721.

The authors are thankful to the anonymous referees for valuable suggestions.

\section{References}

[1] E. Bishop, Foundations of Constructive Analysis, McGraw-Hill, New York, 1967.

[2] L. Boltzmann, Bemrkungen über einige Probleme der mechanischen Wärmtheorie, Wiener Ber. II, 75:62-100, 1877.

[3] D. Dubois and H. Prade, Possibility Theory: An Approach to Computerized Processing of Uncertainty, Plenum Press, New York, 1988.

[4] R. P. Feynman, Statistical Mechanics, W. A. Benjamin, New York, 1972.

[5] R. Feynman, R. Leighton, and M. Sands, The Feynman Lectures on Physics, Addison Wesley, Boston, Massachusetts, 2005.

[6] A. M. Finkelstein, O. Kosheleva, V. Kreinovich, S. A. Starks, and H. T. Nguyen, To properly reflect physicists' reasoning about randomness, we also need a maxitive (possibility) measure, Proceedings of the 2005 IEEE International Conference on Fuzzy Systems FUZZ-IEEE'2005, Reno, Nevada, May 22-25, 2005, pp. 1044-1049.

[7] A. M. Finkelstein, O. Kosheleva, V. Kreinovich, S. A. Starks, and H. T. Nguyen, Use of maxitive (possibility) measures in foundations of physics and description of randomness: case study, Proceedings of the 24th International Conference of the North American Fuzzy Information Processing Society NAFIPS'2005, Ann Arbor, Michigan, June 22-25, 2005, pp. 687-692.

[8] A. M. Finkelstein and V. Kreinovich, Impossibility of hardly possible events: physical consequences, In: Abstracts of the 8th international congress on logic, methodology and philosophy of science, Moscow, 5(2):25-27, 1987.

[9] G. J. Klir and B. Yuan, Fuzzy Sets and Fuzzy Logic: Theory and Applications, Prentice Hall, Upper Saddle River, New Jersey, 1995.

[10] V. Kreinovich, Toward formalizing nonmonotonic reasoning in physics: the use of Kolmogorov complexity and Algorithmic Information Theory to formalize the notions "typically" and "normally", In: L. Sheremetov and M. Alvarado (eds.), Proceedings of the Workshops on Intelligent Computing WIC'04 associated with the Mexican International Conference on Artificial Intelligence MICAI'04, Mexico City, Mexico, April 26-27, 2004, pp. 187-194.

[11] V. Kreinovich, Toward formalizing nonmonotonic reasoning in physics: the use of Kolmogorov complexity, Revista Iberoamericana de Inteligencia Artificial, 41:4-20, 2009.

[12] V. Kreinovich, Negative results of computable analysis disappear if we restrict ourselves to random (or, more generally, typical) inputs, Mathematical Structures and Modeling, 25:100-113, 2012.

[13] V. Kreinovich, Towards formalizing nonmonotonic reasoning in physics: logical approach based on physical induction and its re- 
lation to Kolmogorov complexity. In E. Erdem, J. Lee, Y. Lierler, and D. Pearce (eds.), Correct Reasoning: Essays on Logic-Based AI in Honor of Vladimir Lifschitz, Springer Verlag, Lectures Notes in Computer Science 7265:390-404, 2012.

[14] V. Kreinovich and A. M. Finkelstein, Towards applying computational complexity to foundations of physics, Notes of Mathematical Seminars of St. Petersburg Department of Steklov Institute of Mathematics, 316:63-110, 2004.

[15] V. Kreinovich and A. M. Finkelstein, Towards applying computational complexity to foundations of physics, Journal of Mathematical Sciences, 134:2358-2382, 2006.

[16] V. Kreinovich and O. Kosheleva, Logic of scientific discovery: how physical induction affects what is computable, Proceedings of the International Interdisciplinary Conference Philosophy, Mathematics, Linguistics: Aspects of Interaction 2014 PhML'2014, St. Petersburg, Russia, April 21-25, 2014, pp. 116-127.
[17] M. Li and P. M. B. Vitányi, An Introduction to Kolmogorov Complexity, SpringerVerlag, Berlin, Heidelberg, New York, 2008.

[18] H. T. Nguyen and E. A. Walker, A First Course in Fuzzy Logic, Chapman and Hall/CRC, Boca Raton, Florida, 2006.

[19] H. Rogers, Jr., The Theory of Recursive Functions and Effective Computability, MIT Press, Cambridge, Massachusetts, 1987.

[20] K. Weihrauch, Computable Analysis, Springer Verlag, Berlin, 2000.

[21] L. A. Zadeh, Fuzzy sets, Information and Control, 8:338-353, Elsevier, 1965.

[22] L. Zadeh, Fuzzy sets as a basis for a theory of possibility, Fuzzy Sets and Systems, 1:3-28, 1978 . 